\title{
Lockin thermography for imaging of modulated flow in blood vessels
}

\author{
by D. Wu', H. Hamann ${ }^{2}, A$. Salerno ${ }^{1}$, and G. Busse ${ }^{1}$ \\ 1 Institut fur Kunststoffprufung und Kunststoffkunde, Universităt Stuttgart, Pfaffenwaldring 32, D- \\ 70569 Stuttgart \\ 2 Kreiskrankenhaus Leonberg, Rutesheimer Str. 50, D-71229 Leonberg
}

\begin{abstract}
While conventional thermographic applications to the human body aim at revealing local temperature changes normally related with the beginning of a pathologic process, this paper shows how lockin thermography can be modified in order to meet medical needs in the visualization of functionality of blood vessels.

A compression cuff is used in connection with the lockin system to create a modulation in the blood flow. The amplitude and phase images thus obtained can provide information about the blood flow in the vessels.
\end{abstract}

\section{Introduction}

The idea of nondestructive testing is that an object may be inspected without affecting its integrity or functionality.

This aspect is particularly appreciated if the object under inspection is the human body. Therefore X-rays and ultrasonic inspection as techniques of nondestructive testing have soon found acceptance for medical inspection, where hidden structures (and their defects) can be seen without operating on the person itself.

Nondestructive testing characterizes the inspected object by the way it reacts to a certain kind of input.

In the case of X-ray inspection electromagnetic waves penetrate the body where they are partially absorbed: the resulting image shows how they are attenuated in various regions of the body. Similarly an ultrasonic image reveals hidden boundaries from the reflection of elastic waves on them.

A third kind of wave is being used for nondestructive and remote characterization: thermal waves. They are suited to locate boundaries in near-surface areas, so they combine remote inspection with boundary sensitivity

The purpose of this paper is to show how the natural heating system of the body can be used for thermal wave generation and thereby for remote inspection of internal structures and their functionality.

\section{Thermal waves and lockin thermography}

If heat is periodically injected into material, the resulting oscillating temperature field is described as a thermal wave.

A thermal wave is heavily damped and an important quantity related to it is the thermal diffusion length: this is the distance across which the temperature amplitude of a plane thermal wave decays to $1 / e \approx 37 \%$ of its initial value.

In most technical applications thermal waves are generated by periodical illumination of the object surface. Detection of the resulting temperature oscillation is performed in a remote way by an infrared detector that responds only to the temperature modulation and the variation of its phase across the object. The input to the tested object is remotely injected modulated heat, while the output is phase and magnitude of resulting surface temperature modulation. 
In lockin thermography one can present this local phase shift as an image where color or gray shade represent this angle which can be considered as a delay time of propagation ("phase image").

\section{Internally generated thermal waves}

Instead of injecting thermal waves from outside, they can be generated within the object itself if suitable heating elements are available.

These can be metallic structures which are buried sources of thermal waves if an oscillatory voltage is applied to generate heat by resistive heating. If the distance from the surface is not too large one has a chance to visualize the hidden heating source from the phase field generated on the surface. At low frequencies the thermal diffusion length $\mu$ becomes larger

$$
\mu=\sqrt{2 \alpha / \omega}
$$

where $\alpha$ is thermal diffusivity and $\omega$ the angular frequency of modulation. Therefore the distance from the source to the surface may be larger at lower frequencies.

Besides resistive electrical heating (and other internal sources, e.g. hysteresis heating [1]) there is one more way described already in 1863 by Angstrom: his thermal wave generator was a tube whose temperature was modulated by switching the water flowing through it between hot and cold. In our work we use the internal tube system of the body and modulate the blood flow in it.

\section{Experimental setup}

Temperature control within the body is regulated via the flow in the blood vessels.

The blood vessels themselves can be seen using ultrasonics, which is a contacting method. If the blood flow is modulated in a suitable way, each blood vessel acts like a thermal wave emitter, which can be detected in a remote way on the skin via the resulting phase field. It would be very convenient to use the natural modulation of flow provided by the heart. However, this frequency together with the low thermal conductivity of the body does not allows for enough depth range. Much lower frequencies are needed to obtain images resulting from modulated flow in blood vessels in a depth of several $\mathrm{mm}$.

in terms of the response concept mentioned above, the input is a modulation of blood pressure, the response of the human system is the modulation of blood flow (figure 1) detected via the modulation of temperature on the skin.

Therefore such an image shows selectively only those blood vessels where pressure modulation results in modulated flow providing a map of the functionality of vessels, obtained in a remote way.

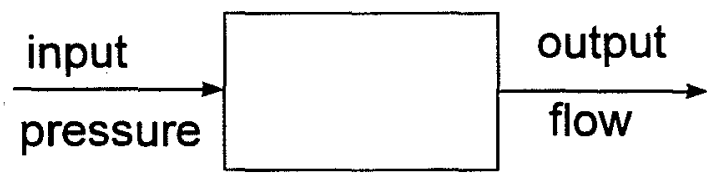

Fig. 1. Blood flow modulation is the response to a modulated pressure input.

The amplitude of the modulation depends on the amount of heat which is proportional to the blood flow, while the phase image displays the total normalized propagation time from the vessel to the surface, so it reveals mainly the depth where the vessel is located (though the wall of the vessel also has importance and might be interesting for detecting deposited material). Also the propagation time of flow itself is involved, which depends on flow resistance.

In one measurement the blood pressure modulation was achieved by computer controlled application of air pressure to a compression cuff around the arm (figure 2). The pressure range was the one of conventional blood pressure measurements.

The resulting field of blood flow modulation was monitored on the forearm. 


\section{http://dx.doi.org/10.21611/qirt.1996.057}

The correlation between image acquisition, modulation frequency and calculation of amplitude and phase image has been described previously $[2,3]$.

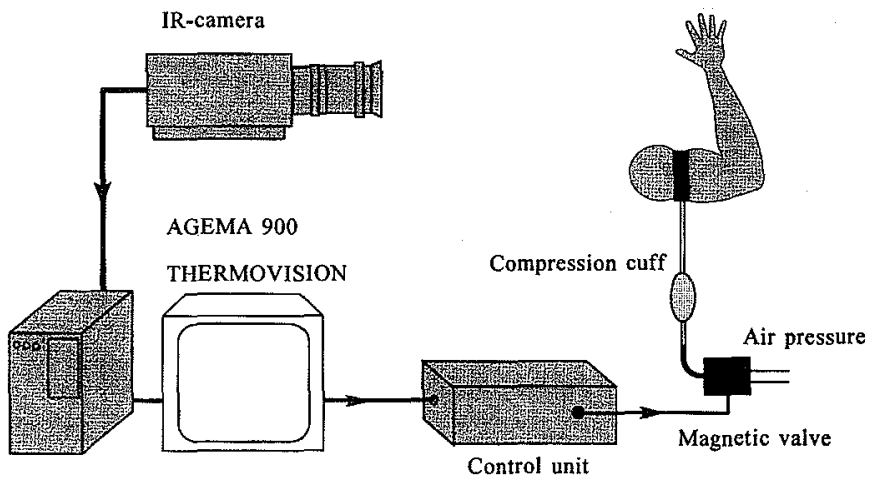

Fig. 2. Experimental setup

\section{Results}

Both the magnitude image and the phase angle images are shown.

Figure 3 shows the amplitude (a) and the phase image (b) of the forearm at $0.03 \mathrm{~Hz}$, while figure 4 was obtained at $0.015 \mathrm{~Hz}$ to give more depth range.

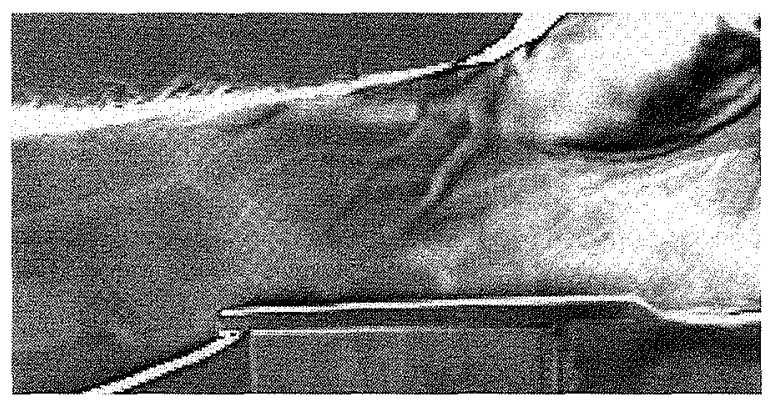

Fig. 3 a. Amplitude lockin thermographic image at $0.03 \mathrm{~Hz}$.

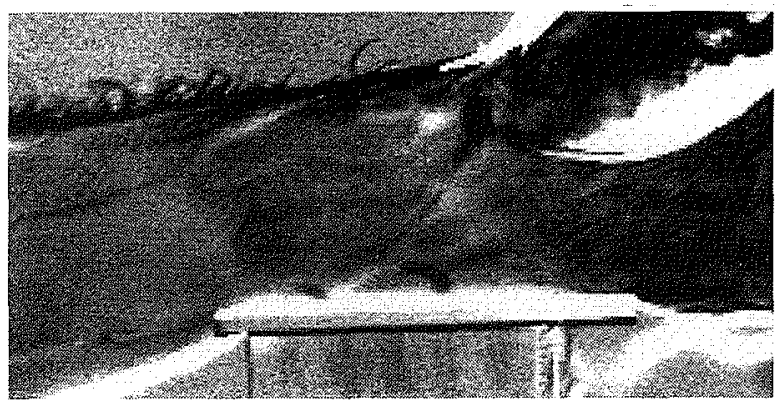

Fig. $3 \mathrm{~b}$. Phase lockin thermographic image at $0.03 \mathrm{~Hz}$. 


\section{http://dx.doi.org/10.21611/qirt.1996.057}

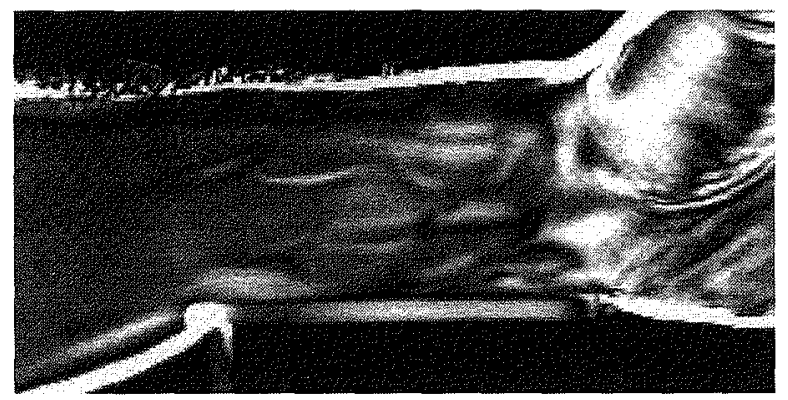

Fig. 4 a. Amplitude lockin thermographic image at $0.015 \mathrm{~Hz}$.

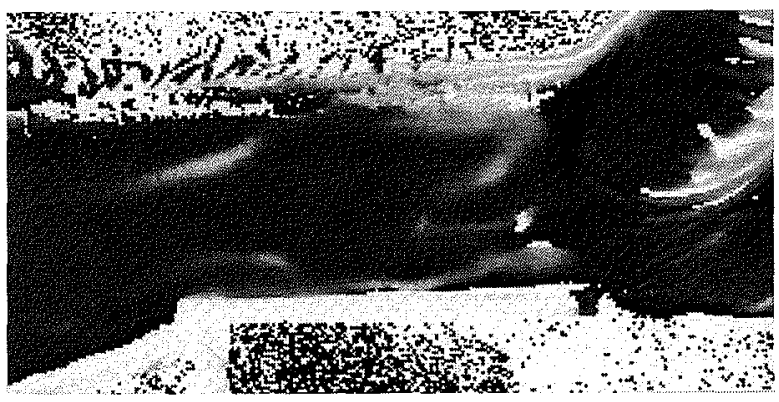

Fig. 4 b. Phase lockin thermographic image at $0.015 \mathrm{~Hz}$.

In the magnitude images the specific contrast mechanism is the modulation depth of temperature on the skin, so it shows selectively those blood vessels whose blood flow responds to the pressure modulation.

The phase image, however, displays the local time delay between the pressure modulation and the resulting temperature modulation at the skin surface. This delay contains the propagation speed of the blood in the vessel and the thermal propagation time from the vessel to the outer surface of the arm.

This is a completely different contrast mechanism as is seen from the phase image at 0.015 $\mathrm{Hz}$, where only certain blood vessels reveal themselves as blood pressure activated heat sources.

\section{Conclusions}

The blood flow in the forearm consequent to a pressure modulation applied to the upper arm has been visualized with lockin thermography.

As compared to conventional thermography showing just temperature fields, this kind of lockin thermography responds only to dynamical processes triggered by a certain input. Therefore it may help to monitor transport processes and the functionality of blood vessels in the body.

\section{Acknowledgments}

A. Salerno is greatly indebted to the Foundation Blanceflor Boncompagni Ludovisi Bildt for the fellowship that allowed him to take part into the present work. 


\section{REFERENCES}

[1] RANTALA (J.), WU (D.), BUSSE (G.); Amplitude Modulated Lockin Vibrothermography for NDE of Polymers and Composites - Research in Nondestructive Evaluation (1996) 7 ,
pp.215-228. [2] BUSSE (G.), WU (D.), and KARPEN (W.); Thermal wave imaging with phase sensitive modulated thermography; J. Appl. Phys., 71, 8, (1992) pp. 3962-3965.

[3] WU (D.), BUSSE (G.); Zerstörungsfreie phasenempfindliche Modulationsthermographie; Materialprüfung 36 (1994) 10, pp. 393-399. 